\title{
Isoflurane as an Accurate Negative Mode Calibrant for Ion Mobility Spectrometry
}

\author{
Brian C. Hauck, ${ }^{1, \text { a) }}$ Charles S. Harden, ${ }^{1}$ Vincent M. McHugh, ${ }^{2}$
}

1. Science and Technology Corporation (STC) - Support to DEVCOM CBC, Belcamp, MD 21017

2. U.S. Army Combat Capabilities Development Command Chemical Biological Center (DEVCOM CBC), Aberdeen Proving Ground, MD 21010

a) Present Address: U.S. Army Combat Capabilities Development Command Chemical Biological Center (DEVCOM CBC), Aberdeen Proving Ground, MD 21010

\section{Table of Contents}

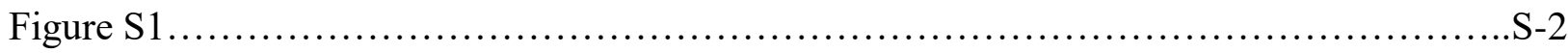

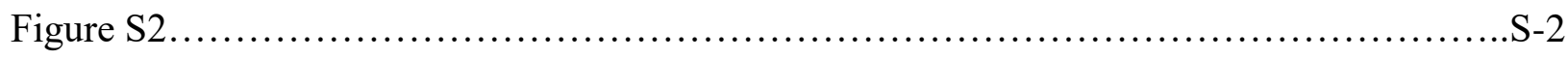

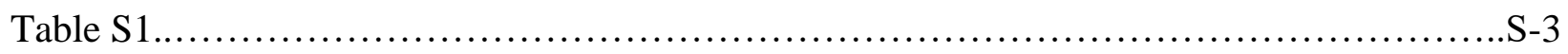

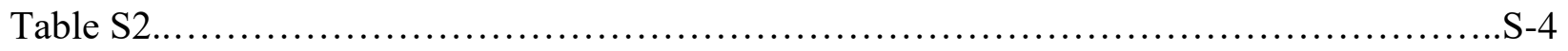

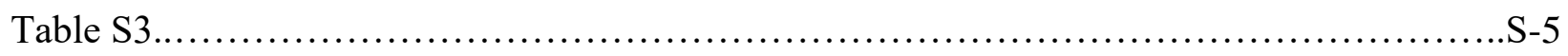

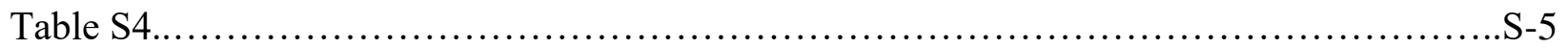

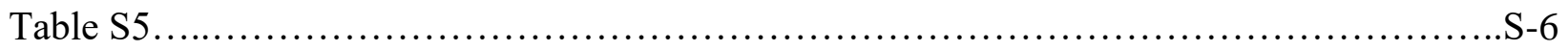

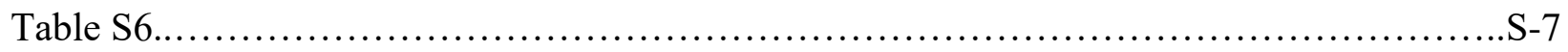

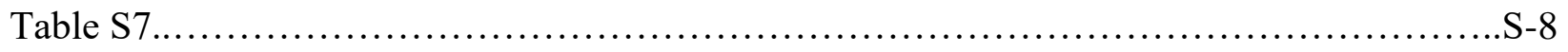

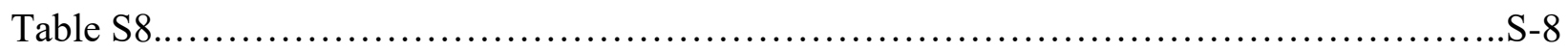




\section{Supplemental Figures}

The figures created from accurate reduced ion mobility $\left(K_{0}\right)$ values measured in standalone ion mobility spectrometry (IMS) mode, corroborating the values measured in ion mobility-mass spectrometry (IMMS) mode in Figures 4 and 6, are shown in Figures S2 and S3, respectively.

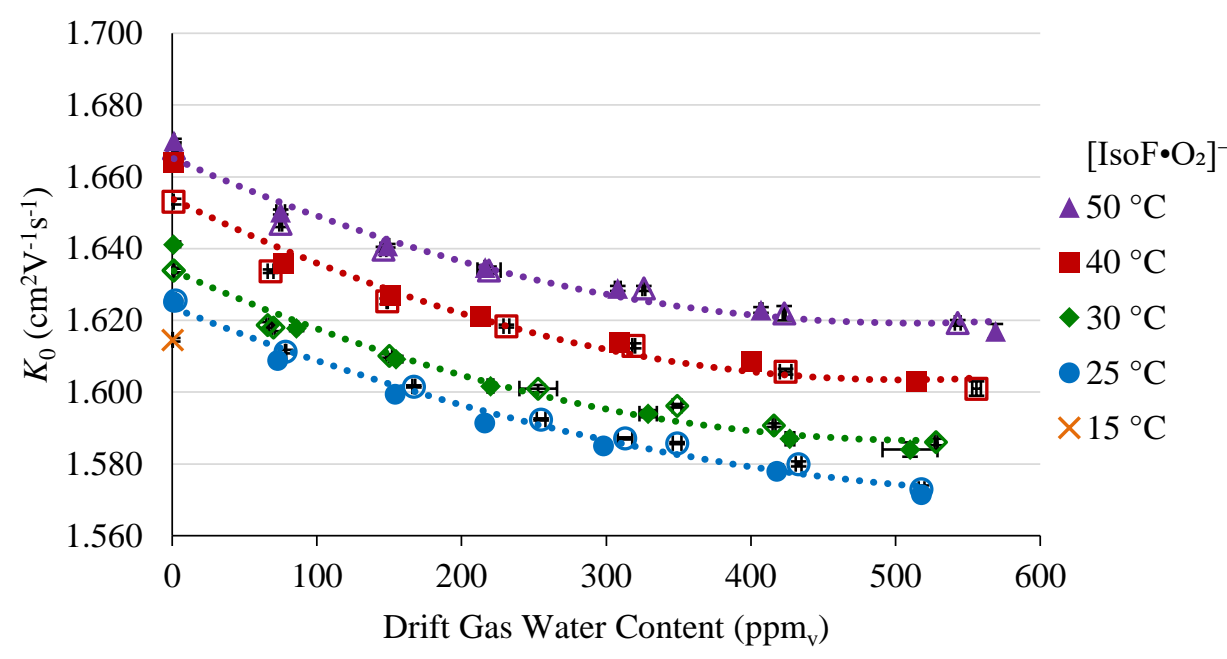

Figure S1. Effect of drift gas water concentration on the $K_{0}$ value for the oxygen adductof isoflurane, $\left[\mathrm{IsoF} \bullet \mathrm{O}_{2}\right]^{-}$, in standalone IMS mode at five drift gas temperatures under ammonia doped (solid marker) and undoped (open marker) conditions.

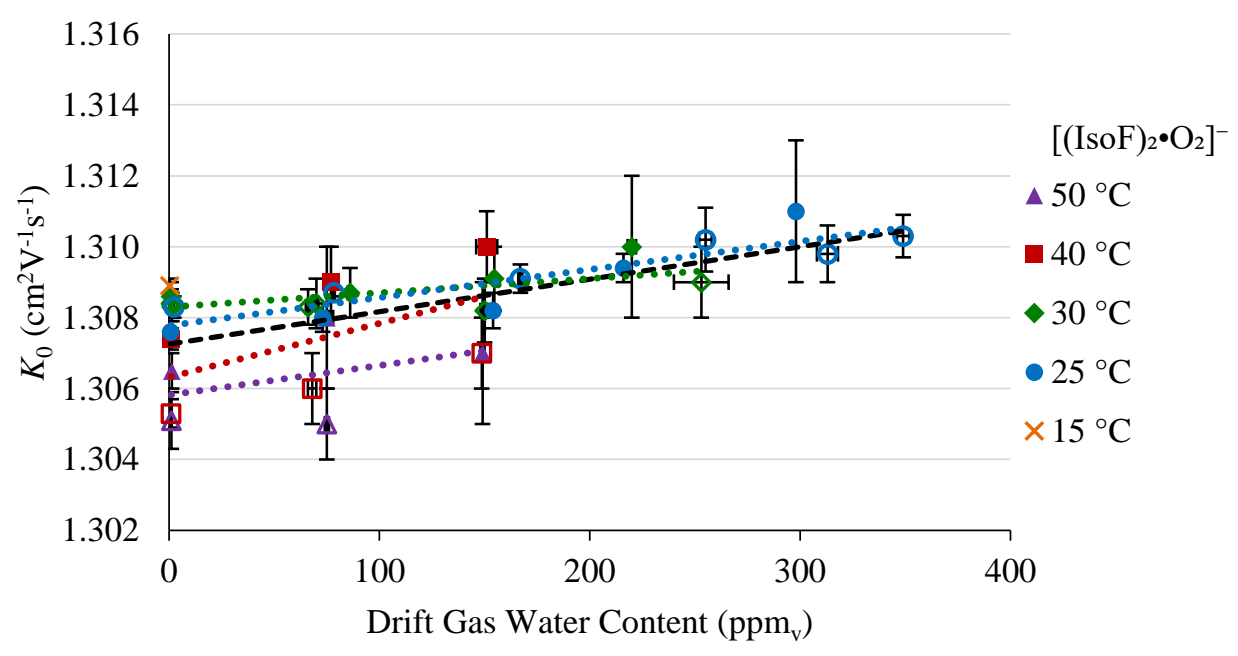

Figure S2. Effect of drift gas water concentration on the $K_{0}$ value for the oxygen-bound dimer of isoflurane, $\left[(\mathrm{IsoF})_{2} \bullet \mathrm{O}_{2}\right]^{-}$, in standalone IMS mode at five drift gas temperatures under ammoniadoped (solid marker) and undoped (open marker) conditions. 


\section{Accurate $K_{0}$ Values of Isoflurane}

The accurate $K_{0}$ values for the isoflurane (IsoF) product ions (used to create Figures 4-7, S-2, and S-3) are shown in Tables S-1 through S-8. A low-intensity nitrate adduct of isoflurane, $\left[\mathrm{IsoF} \bullet \mathrm{NO}_{2}\right]^{-}$, product ion peak was observed in IMMS mode when the Bradbury- Nielson ion gates were left open. $K_{0}$ values for the [IsoF $\left.\bullet \mathrm{NO}_{2}\right]^{-}$product ion are not reported because of the great amount of time that would be required to collect sufficient signal. Each $K_{0}$ value is an average of at least three measurements, and precision is represented by error bars in the figures (when not visible, error bars are hidden by the marker).

Table S1. $K_{0}$ Values for [IsoF• $\left.\mathrm{O}_{2}\right]^{-}$and $\left[(\mathrm{IsoF})_{2} \bullet \mathrm{O}_{2}\right]^{-}$on IMS-TOFMSat $280.1 \mathrm{~V} / \mathrm{cm}$ as a Function of Drift Gas Water Concentration at Four Drift Gas Temperatures under Undoped Conditions

\begin{tabular}{|c|c|c|c|}
\hline \multirow{2}{*}{$\begin{array}{c}\text { Average } \\
\text { Drift Gas } \\
\text { Temperature } \\
\left({ }^{\circ} \mathbf{C}\right)\end{array}$} & \multirow{2}{*}{$\begin{array}{c}\text { Average } \\
\text { Drift Gas } \\
\text { Water } \\
\text { Concentration } \\
\left(\operatorname{ppm}_{\mathrm{v}} \mathrm{H}_{2} \mathrm{O}\right)\end{array}$} & \multicolumn{2}{|c|}{$\begin{array}{c}K_{0} \text { Value } \\
\text { (Precision, Accuracy) } \\
\left(\mathrm{cm}^{2} \mathbf{V}^{-1} \mathbf{s}^{-1}\right)\end{array}$} \\
\hline & & $\begin{array}{c}\left.{ }^{[I s o F} \bullet \mathrm{O}_{2}\right]^{-} \\
m / z 216,218\end{array}$ & $\begin{array}{l}{\left[(\mathrm{IsoF})_{2} \bullet \mathrm{O}_{2}\right]^{-}} \\
m / z, \mathbf{4 0 0}, \mathbf{4 0 2}\end{array}$ \\
\hline \multirow{7}{*}{$25.00 \pm 0.08$} & $1.07 \pm 0.02$ & $1.630( \pm 0.001, \pm 0.002)$ & $1.308( \pm 0.001, \pm 0.001)$ \\
\hline & $75 \pm 2$ & $1.616( \pm 0.001, \pm 0.002)$ & $1.3064( \pm 0.0001, \pm 0.001)$ \\
\hline & $160 \pm 2$ & $1.603( \pm 0.001, \pm 0.002)$ & $1.307( \pm 0.001, \pm 0.001)$ \\
\hline & $250 \pm 7$ & $1.5957( \pm 0.0006, \pm 0.002)$ & N/A \\
\hline & $338 \pm 8$ & $1.589( \pm 0.002, \pm 0.002)$ & N/A \\
\hline & $425 \pm 10$ & $1.584( \pm 0.002, \pm 0.002)$ & N/A \\
\hline & $510 \pm 5$ & $1.581( \pm 0.002, \pm 0.002)$ & N/A \\
\hline \multirow{7}{*}{$30.02 \pm 0.03$} & $1.00 \pm 0.02$ & $1.6404( \pm 0.0005, \pm 0.002)$ & $1.3092( \pm 0.0004, \pm 0.001)$ \\
\hline & $65 \pm 2$ & $1.624( \pm 0.001, \pm 0.002)$ & $1.308( \pm 0.002, \pm 0.001)$ \\
\hline & $150 \pm 1$ & $1.615( \pm 0.002, \pm 0.002)$ & $1.309( \pm 0.001, \pm 0.001)$ \\
\hline & $226 \pm 6$ & $1.6063( \pm 0.0008, \pm 0.002)$ & N/A \\
\hline & $358 \pm 6$ & $1.5955( \pm 0.0008, \pm 0.002)$ & N/A \\
\hline & $416 \pm 5$ & $1.5923( \pm 0.0006, \pm 0.002)$ & N/A \\
\hline & $526 \pm 4$ & $1.5880( \pm 0.0005, \pm 0.002)$ & N/A \\
\hline \multirow{7}{*}{$40.01 \pm 0.04$} & $0.84 \pm 0.01$ & $1.6544( \pm 0.0008, \pm 0.002)$ & $1.307( \pm 0.002, \pm 0.001)$ \\
\hline & $64 \pm 2$ & $1.6370( \pm 0.0003, \pm 0.002)$ & $1.307( \pm 0.001, \pm 0.001)$ \\
\hline & $146 \pm 1$ & $1.6281( \pm 0.0008, \pm 0.002)$ & $1.3046( \pm 0.0004, \pm 0.001)$ \\
\hline & $227 \pm 8$ & $1.6225( \pm 0.0006, \pm 0.002)$ & N/A \\
\hline & $296 \pm 1$ & $1.617( \pm 0.001, \pm 0.002)$ & N/A \\
\hline & $419 \pm 3$ & $1.611( \pm 0.002, \pm 0.002)$ & N/A \\
\hline & $513 \pm 5$ & $1.606( \pm 0.001, \pm 0.002)$ & N/A \\
\hline \multirow{7}{*}{$50.01 \pm 0.04$} & $1.00 \pm 0.01$ & $1.6712( \pm 0.0005, \pm 0.002)$ & $1.306( \pm 0.002, \pm 0.001)$ \\
\hline & $72 \pm 3$ & $1.651( \pm 0.001, \pm 0.002)$ & $1.308( \pm 0.002, \pm 0.001)$ \\
\hline & $149 \pm 1$ & $1.6427( \pm 0.0002, \pm 0.002)$ & N/A \\
\hline & $219.7 \pm 0.2$ & $1.636( \pm 0.002, \pm 0.002)$ & N/A \\
\hline & $313 \pm 2$ & $1.631( \pm 0.002, \pm 0.002)$ & N/A \\
\hline & $422 \pm 3$ & $1.626( \pm 0.002, \pm 0.002)$ & N/A \\
\hline & $546 \pm 2$ & $1.621( \pm 0.002, \pm 0.002)$ & N/A \\
\hline
\end{tabular}

N/A, not applicable. 
Table S2. $K_{0}$ Values for [IsoF• $\left.\mathrm{O}_{2}\right]^{-}$and $\left[(\mathrm{IsoF})_{2} \bullet \mathrm{O}_{2}\right]^{-}$on IMS-TOFMS at $280.1 \mathrm{~V} / \mathrm{cm}$ as a Function of Drift Gas Water Concentration at Five Drift Gas Temperatures under AmmoniaDoped Conditions

\begin{tabular}{|c|c|c|c|}
\hline \multirow{2}{*}{$\begin{array}{c}\text { Average } \\
\text { Drift Gas } \\
\text { Temperature } \\
\left({ }^{\circ} \mathbf{C}\right)\end{array}$} & \multirow{2}{*}{$\begin{array}{c}\text { Average } \\
\text { Drift Gas } \\
\text { Water } \\
\text { Concentration } \\
\left(\operatorname{ppm}_{\mathbf{v}} \mathbf{H}_{2} \mathrm{O}\right)\end{array}$} & \multicolumn{2}{|c|}{$\begin{array}{c}K_{0} \text { Value } \\
\text { (Precision, Accuracy) } \\
\left(\mathrm{cm}^{2} \mathbf{V}^{-1} \mathbf{s}^{-1}\right)\end{array}$} \\
\hline & & $\begin{array}{c}{\left[\mathrm{IsoF} \cdot \mathrm{O}_{2}\right]^{-}} \\
m / z, 216,218\end{array}$ & $\begin{array}{l}{\left[(\mathrm{IsoF})_{2} \bullet \mathrm{O}_{2}\right]^{-}} \\
m / z \mathbf{4 0 0 ,}, \mathbf{4 0 2}\end{array}$ \\
\hline $15.0 \pm 0.3$ & $0.389 \pm 0.006$ & $1.618( \pm 0.002, \pm 0.002)$ & $1.310( \pm 0.002, \pm 0.001)$ \\
\hline \multirow{7}{*}{$25.00 \pm 0.08$} & $1.01 \pm 0.02$ & $1.6295( \pm 0.0006, \pm 0.002)$ & $1.3101( \pm 0.0003, \pm 0.001)$ \\
\hline & $72 \pm 1$ & $1.615( \pm 0.001, \pm 0.002)$ & $1.309( \pm 0.001, \pm 0.001)$ \\
\hline & $142 \pm 3$ & $1.6044( \pm 0.0003, \pm 0.002)$ & $1.3084( \pm 0.0006, \pm 0.001)$ \\
\hline & $213 \pm 4$ & $1.5971( \pm 0.0007, \pm 0.002)$ & $1.309( \pm 0.002, \pm 0.001)$ \\
\hline & $307 \pm 3$ & $1.5898( \pm 0.0005, \pm 0.002)$ & N/A \\
\hline & $399 \pm 3$ & $1.582( \pm 0.002, \pm 0.002)$ & N/A \\
\hline & $503 \pm 4$ & $1.573( \pm 0.002, \pm 0.002)$ & N/A \\
\hline \multirow{7}{*}{$30.07 \pm 0.03$} & $0.314 \pm 0.003$ & $1.651( \pm 0.002, \pm 0.002)$ & $1.309( \pm 0.001, \pm 0.001)$ \\
\hline & $80 \pm 2$ & $1.6257( \pm 0.0006, \pm 0.002)$ & $1.3075( \pm 0.0009, \pm 0.001)$ \\
\hline & $141 \pm 1$ & $1.6135( \pm 0.0008, \pm 0.002)$ & $1.3086( \pm 0.0008, \pm 0.001)$ \\
\hline & $225 \pm 1$ & $1.6060( \pm 0.0009, \pm 0.002)$ & $1.313( \pm 0.002, \pm 0.001)$ \\
\hline & $323 \pm 2$ & $1.5980( \pm 0.0004, \pm 0.002)$ & N/A \\
\hline & $437 \pm 17$ & $1.5906( \pm 0.0001, \pm 0.002)$ & N/A \\
\hline & $518 \pm 6$ & $1.5853( \pm 0.0009, \pm 0.002)$ & N/A \\
\hline \multirow{7}{*}{$40.04 \pm 0.02$} & $0.83 \pm 0.01$ & $1.6756( \pm 0.0005, \pm 0.002)$ & $1.3069( \pm 0.0001, \pm 0.001)$ \\
\hline & $68 \pm 7$ & $1.6415( \pm 0.0007, \pm 0.002)$ & $1.307( \pm 0.001, \pm 0.001)$ \\
\hline & $141 \pm 1$ & $1.632( \pm 0.001, \pm 0.002)$ & $1.310( \pm 0.001, \pm 0.001)$ \\
\hline & $216 \pm 1$ & $1.625( \pm 0.001, \pm 0.002)$ & N/A \\
\hline & $296 \pm 2$ & $1.619( \pm 0.002, \pm 0.002)$ & N/A \\
\hline & $417 \pm 1$ & $1.6104( \pm 0.0006, \pm 0.002)$ & N/A \\
\hline & $512 \pm 16$ & $1.6073( \pm 0.0006, \pm 0.002)$ & N/A \\
\hline \multirow{7}{*}{$50.00 \pm 0.03$} & $0.25 \pm 0.01$ & $1.689( \pm 0.001, \pm 0.002)$ & $1.306( \pm 0.001, \pm 0.001)$ \\
\hline & $66 \pm 6$ & $1.657( \pm 0.001, \pm 0.002)$ & $1.3095( \pm 0.0005, \pm 0.001)$ \\
\hline & $143 \pm 1$ & $1.6466( \pm 0.0007, \pm 0.002)$ & N/A \\
\hline & $215 \pm 1$ & $1.638( \pm 0.001, \pm 0.002)$ & N/A \\
\hline & $303 \pm 2$ & $1.635( \pm 0.002, \pm 0.002)$ & N/A \\
\hline & $425 \pm 8$ & $1.6282( \pm 0.0005, \pm 0.002)$ & N/A \\
\hline & $506 \pm 9$ & $1.624( \pm 0.001, \pm 0.002)$ & N/A \\
\hline
\end{tabular}

N/A, not applicable. 
Table S3. $K_{0}$ Values for [IsoF $\left.\bullet \mathrm{CO}_{3}\right]^{-}(\mathrm{m} / \mathrm{z}, 244,246)$ on IMS-TOFMS at $280.1 \mathrm{~V} / \mathrm{cm}$ as a Function of Drift Gas Water Concentration at Four Drift Gas Temperatures under Undoped Conditions

\begin{tabular}{|c|c|c|}
\hline $\begin{array}{c}\text { Average } \\
\text { Drift Gas } \\
\text { Temperature } \\
\left({ }^{\circ} \mathbf{C}\right)\end{array}$ & $\begin{array}{c}\text { Average } \\
\text { Drift Gas } \\
\text { Water } \\
\text { Concentration } \\
\left(\mathbf{p p m}_{\mathrm{v}} \mathrm{H}_{2} \mathrm{O}\right)\end{array}$ & $\begin{array}{c}K_{0} \text { Value } \\
\text { (Precision, Accuracy) } \\
\left(\mathrm{cm}^{2} \mathbf{V}^{-1} \mathbf{s}^{-1}\right)\end{array}$ \\
\hline \multirow{3}{*}{$25.00 \pm 0.08$} & $1.07 \pm 0.02$ & $1.610( \pm 0.001, \pm 0.002)$ \\
\hline & $75 \pm 2$ & $1.6025( \pm 0.0006, \pm 0.002)$ \\
\hline & $160 \pm 2$ & $1.601( \pm 0.002, \pm 0.002)$ \\
\hline \multirow{3}{*}{$30.02 \pm 0.03$} & $1.00 \pm 0.02$ & $1.613( \pm 0.002, \pm 0.002)$ \\
\hline & $65 \pm 2$ & $1.611( \pm 0.002, \pm 0.002)$ \\
\hline & $150 \pm 1$ & $1.605( \pm 0.002, \pm 0.002)$ \\
\hline \multirow{3}{*}{$40.01 \pm 0.04$} & $0.84 \pm 0.01$ & $1.6188( \pm 0.0007, \pm 0.002)$ \\
\hline & $64 \pm 2$ & $1.617( \pm 0.002, \pm 0.002)$ \\
\hline & $146 \pm 1$ & $1.616( \pm 0.002, \pm 0.002)$ \\
\hline \multirow{2}{*}{$50.01 \pm 0.04$} & $1.00 \pm 0.01$ & $1.6245( \pm 0.0007, \pm 0.002)$ \\
\hline & $72 \pm 3$ & $1.625( \pm 0.002, \pm 0.002)$ \\
\hline
\end{tabular}

Table S4. $K_{0}$ Values for [IsoF• $\left.\mathrm{CO}_{3}\right]^{-}(\mathrm{m} / z$ 244, 246) on IMS-TOFMS at $280.1 \mathrm{~V} / \mathrm{cm}$ as a Function of Drift Gas Water Concentration at Five Drift Gas Temperatures under AmmoniaDoped Conditions

\begin{tabular}{|c|c|c|}
\hline $\begin{array}{c}\text { Average } \\
\text { Drift Gas } \\
\text { Temperature } \\
\left({ }^{\circ} \mathbf{C}\right)\end{array}$ & $\begin{array}{c}\text { Average } \\
\text { Drift Gas } \\
\text { Water Concentration } \\
\left(\mathbf{p p m}_{\mathbf{v}} \mathbf{H}_{2} \mathrm{O}\right)\end{array}$ & $\begin{array}{c}K_{0} \text { Value } \\
\text { (Precision, Accuracy) } \\
\left(\mathrm{cm}^{2} \mathbf{V}^{-1} \mathbf{s}^{-1}\right)\end{array}$ \\
\hline $15.0 \pm 0.3$ & $0.389 \pm 0.006$ & $1.601( \pm 0.001, \pm 0.002)$ \\
\hline \multirow{3}{*}{$25.00 \pm 0.08$} & $1.01 \pm 0.02$ & $1.609( \pm 0.001, \pm 0.002)$ \\
\hline & $72 \pm 1$ & $1.604( \pm 0.002, \pm 0.002)$ \\
\hline & $142 \pm 3$ & $1.5998( \pm 0.0005, \pm 0.002)$ \\
\hline \multirow{3}{*}{$30.07 \pm 0.03$} & $0.314 \pm 0.003$ & $1.6161( \pm 0.0005, \pm 0.002)$ \\
\hline & $80 \pm 2$ & $1.610( \pm 0.001, \pm 0.002)$ \\
\hline & $141 \pm 1$ & $1.605( \pm 0.002, \pm 0.002)$ \\
\hline \multirow{2}{*}{$40.04 \pm 0.02$} & $0.83 \pm 0.01$ & $1.620( \pm 0.001, \pm 0.002)$ \\
\hline & $68 \pm 7$ & $1.619( \pm 0.001, \pm 0.002)$ \\
\hline \multirow{2}{*}{$50.00 \pm 0.03$} & $0.25 \pm 0.01$ & $1.627( \pm 0.001, \pm 0.002)$ \\
\hline & $66 \pm 6$ & $1.625( \pm 0.002, \pm 0.002)$ \\
\hline
\end{tabular}


Table S5. $K_{0}$ Values for First Monomer and Dimer Peaks, Corresponding to [IsoF $\left.\bullet \mathrm{O}_{2}\right]^{-}$and $\left[(\mathrm{IsoF})_{2} \bullet \mathrm{O}_{2}\right]^{-}$, Respectively, on IMS Faraday Plate at $280.1 \mathrm{~V} / \mathrm{cm}$ as a Function of Drift Gas Water Concentration at Four Drift Gas Temperatures under Undoped Conditions

\begin{tabular}{|c|c|c|c|}
\hline \multirow{2}{*}{$\begin{array}{c}\text { Average } \\
\text { Drift Gas } \\
\text { Temperature } \\
\left({ }^{\circ} \mathbf{C}\right)\end{array}$} & \multirow{2}{*}{$\begin{array}{c}\text { Average } \\
\text { Drift Gas } \\
\text { Water Concentration } \\
\left(\mathbf{p p m}_{\mathrm{v}} \mathrm{H}_{\mathbf{2}} \mathrm{O}\right)\end{array}$} & \multicolumn{2}{|c|}{$\begin{array}{c}K_{0} \text { Value } \\
\text { (Precision, Accuracy) } \\
\left(\mathrm{cm}^{2} \mathbf{V}^{-1} \mathbf{s}^{-1}\right)\end{array}$} \\
\hline & & $\begin{array}{c}\text { Monomer Peak } 1 \\
{\left[\mathrm{IsoF} \bullet \mathrm{O}_{2}\right]^{-}}\end{array}$ & $\begin{array}{c}\text { Dimer Peak } \\
{\left[(\text { IsoF })_{2} \bullet \mathrm{O}_{2}\right]^{-}}\end{array}$ \\
\hline \multirow{8}{*}{$25.00 \pm 0.08$} & $2.248 \pm 0.004$ & $1.6256( \pm 0.0002, \pm 0.002)$ & $1.3083( \pm 0.0003, \pm 0.001)$ \\
\hline & $78.4 \pm 0.4$ & $1.6113( \pm 0.0005, \pm 0.002)$ & $1.3087( \pm 0.0001, \pm 0.001)$ \\
\hline & $167 \pm 1$ & $1.6016( \pm 0.0003, \pm 0.002)$ & $1.3091( \pm 0.0004, \pm 0.001)$ \\
\hline & $255 \pm 3$ & $1.5924( \pm 0.0003, \pm 0.002)$ & $1.3102( \pm 0.0009, \pm 0.001)$ \\
\hline & $313 \pm 5$ & $1.5871( \pm 0.0003, \pm 0.002)$ & $1.3098( \pm 0.0008, \pm 0.001)$ \\
\hline & $349 \pm 3$ & $1.5858( \pm 0.0003, \pm 0.002)$ & $1.3103( \pm 0.0006, \pm 0.001)$ \\
\hline & $433 \pm 2$ & $1.5800( \pm 0.0007, \pm 0.002)$ & N/A \\
\hline & $518 \pm 2$ & $1.573( \pm 0.001, \pm 0.002)$ & N/A \\
\hline \multirow{9}{*}{$30.02 \pm 0.03$} & $0.99 \pm 0.02$ & $1.6339( \pm 0.0008, \pm 0.002)$ & $1.3084( \pm 0.0004, \pm 0.001)$ \\
\hline & $1.32 \pm 0.01$ & $1.6339( \pm 0.0005, \pm 0.002)$ & $1.3083( \pm 0.0004, \pm 0.001)$ \\
\hline & $66 \pm 1$ & $1.6187( \pm 0.0008, \pm 0.002)$ & $1.3083( \pm 0.0005, \pm 0.001)$ \\
\hline & $70 \pm 1$ & $1.618( \pm 0.001, \pm 0.002)$ & $1.3084( \pm 0.0007, \pm 0.001)$ \\
\hline & $150 \pm 2$ & $1.6101( \pm 0.0005, \pm 0.002)$ & $1.3082( \pm 0.0009, \pm 0.001)$ \\
\hline & $253 \pm 13$ & $1.601( \pm 0.001, \pm 0.002)$ & $1.309( \pm 0.001, \pm 0.001)$ \\
\hline & $349 \pm 3$ & $1.5962( \pm 0.0005, \pm 0.002)$ & N/A \\
\hline & $416 \pm 1$ & $1.5908( \pm 0.0005, \pm 0.002)$ & N/A \\
\hline & $528 \pm 1$ & $1.5861( \pm 0.0009, \pm 0.002)$ & N/A \\
\hline \multirow{7}{*}{$40.00 \pm 0.03$} & $1.00 \pm 0.01$ & $1.6531( \pm 0.0008, \pm 0.002)$ & $1.3053( \pm 0.0004, \pm 0.001)$ \\
\hline & $68 \pm 2$ & $1.6337( \pm 0.0005, \pm 0.002)$ & $1.306( \pm 0.001, \pm 0.001)$ \\
\hline & $148.6 \pm 0.4$ & $1.6253( \pm 0.0008, \pm 0.002)$ & $1.307( \pm 0.001, \pm 0.001)$ \\
\hline & $231 \pm 2$ & $1.6184( \pm 0.0004, \pm 0.002)$ & N/A \\
\hline & $319 \pm 1$ & $1.6129( \pm 0.0007, \pm 0.002)$ & N/A \\
\hline & $424 \pm 4$ & $1.6057( \pm 0.0008, \pm 0.002)$ & N/A \\
\hline & $556 \pm 3$ & $1.601( \pm 0.002, \pm 0.002)$ & N/A \\
\hline \multirow{7}{*}{$50.01 \pm 0.04$} & $1.06 \pm 0.02$ & $1.668( \pm 0.001, \pm 0.002)$ & $1.3051( \pm 0.0008, \pm 0.001)$ \\
\hline & $75 \pm 1$ & $1.6469( \pm 0.0009, \pm 0.002)$ & $1.305( \pm 0.001, \pm 0.001)$ \\
\hline & $146 \pm 2$ & $1.6398( \pm 0.0007, \pm 0.002)$ & N/A \\
\hline & $219 \pm 8$ & $1.634( \pm 0.001, \pm 0.002)$ & N/A \\
\hline & $326 \pm 1$ & $1.6289( \pm 0.0007, \pm 0.002)$ & N/A \\
\hline & $423 \pm 1$ & $1.622( \pm 0.002, \pm 0.002)$ & N/A \\
\hline & $543 \pm 2$ & $1.6193( \pm 0.0008, \pm 0.002)$ & N/A \\
\hline
\end{tabular}

N/A, not applicable. 
Table S6. $K_{0}$ Values for First Monomer and Dimer Peaks, Corresponding to [IsoF $\left.\bullet \mathrm{O}_{2}\right]^{-}$and [(IsoF $\left.)_{2} \bullet \mathrm{O}_{2}\right]^{-}$, Respectively, on IMS Faraday Plate at $280.2 \mathrm{~V} / \mathrm{cm}$ as a Function of Drift Gas Water Concentration at Five Drift Gas Temperatures under Ammonia-Doped Conditions

\begin{tabular}{|c|c|c|c|}
\hline \multirow{2}{*}{$\begin{array}{c}\text { Average } \\
\text { Drift Gas } \\
\text { Temperature } \\
\left({ }^{\circ} \mathbf{C}\right)\end{array}$} & \multirow{2}{*}{$\begin{array}{c}\text { Average } \\
\text { Drift Gas } \\
\text { Water } \\
\text { Concentration } \\
\left(\mathbf{p p m}_{\mathrm{v}} \mathrm{H}_{2} \mathrm{O}\right) \\
\end{array}$} & \multicolumn{2}{|c|}{$\begin{array}{c}K_{0} \text { Value } \\
\text { (Precision, Accuracy) } \\
\left(\mathrm{cm}^{2} \mathbf{V}^{-1} \mathbf{s}^{-1}\right)\end{array}$} \\
\hline & & $\begin{array}{c}\text { Monomer Peak } 1 \\
{\left[\mathrm{IsoF} \cdot \mathrm{O}_{2}\right]^{-}}\end{array}$ & $\begin{array}{c}\text { Dimer Peak } \\
{\left[(\text { IsoF })_{2} \bullet \mathbf{O}_{2}\right]^{-}}\end{array}$ \\
\hline $15.0 \pm 0.3$ & $0.394 \pm 0.007$ & $1.6146( \pm 0.0005, \pm 0.002)$ & $1.3089( \pm 0.0002, \pm 0.001)$ \\
\hline \multirow{7}{*}{$25.00 \pm 0.08$} & $0.799 \pm 0.001$ & $1.6251( \pm 0.0001, \pm 0.002)$ & $1.3076( \pm 0.0003, \pm 0.001)$ \\
\hline & $73 \pm 3$ & $1.6089( \pm 0.0005, \pm 0.002)$ & $1.3080( \pm 0.0004, \pm 0.001)$ \\
\hline & $154 \pm 1$ & $1.5995( \pm 0.0001, \pm 0.002)$ & $1.3082( \pm 0.0005, \pm 0.001)$ \\
\hline & $216 \pm 1$ & $1.5915( \pm 0.0006, \pm 0.002)$ & $1.3094( \pm 0.0004, \pm 0.001)$ \\
\hline & $298 \pm 1$ & $1.5851( \pm 0.0006, \pm 0.002)$ & $1.311( \pm 0.002, \pm 0.001)$ \\
\hline & $418 \pm 4$ & $1.578( \pm 0.001, \pm 0.002)$ & N/A \\
\hline & $518 \pm 1$ & $1.5715( \pm 0.0006, \pm 0.002)$ & N/A \\
\hline \multirow{7}{*}{$30.07 \pm 0.03$} & $0.99 \pm 0.08$ & $1.6412( \pm 0.0008, \pm 0.002)$ & $1.3086( \pm 0.0004, \pm 0.001)$ \\
\hline & $86 \pm 1$ & $1.6178( \pm 0.0008, \pm 0.002)$ & $1.3087( \pm 0.0007, \pm 0.001)$ \\
\hline & $154.6 \pm 0.3$ & $1.6092( \pm 0.0009, \pm 0.002)$ & $1.3091( \pm 0.0009, \pm 0.001)$ \\
\hline & $220 \pm 2$ & $1.6017( \pm 0.0004, \pm 0.002)$ & $1.310( \pm 0.002, \pm 0.001)$ \\
\hline & $329 \pm 6$ & $1.594( \pm 0.001, \pm 0.002)$ & N/A \\
\hline & $427 \pm 3$ & $1.5870( \pm 0.0005, \pm 0.002)$ & N/A \\
\hline & $510 \pm 19$ & $1.584( \pm 0.002, \pm 0.002)$ & N/A \\
\hline \multirow{7}{*}{$40.04 \pm 0.02$} & $0.955 \pm 0.004$ & $1.664( \pm 0.001, \pm 0.002)$ & $1.3074( \pm 0.0003, \pm 0.001)$ \\
\hline & $77 \pm 2$ & $1.6358( \pm 0.0006, \pm 0.002)$ & $1.309( \pm 0.001, \pm 0.001)$ \\
\hline & $151 \pm 5$ & $1.627( \pm 0.001, \pm 0.002)$ & $1.310( \pm 0.001, \pm 0.001)$ \\
\hline & $213 \pm 1$ & $1.6211( \pm 0.0006, \pm 0.002)$ & N/A \\
\hline & $309.2 \pm 0.3$ & $1.614( \pm 0.001, \pm 0.002)$ & N/A \\
\hline & $400 \pm 4$ & $1.6086( \pm 0.0004, \pm 0.002)$ & N/A \\
\hline & $514 \pm 2$ & $1.603( \pm 0.001, \pm 0.002)$ & N/A \\
\hline \multirow{7}{*}{$50.00 \pm 0.03$} & $1.23 \pm 0.01$ & $1.6701( \pm 0.0005, \pm 0.002)$ & $1.3065( \pm 0.0005, \pm 0.001)$ \\
\hline & $75 \pm 3$ & $1.6502( \pm 0.0007, \pm 0.002)$ & $1.308( \pm 0.002, \pm 0.001)$ \\
\hline & $149 \pm 1$ & $1.6408( \pm 0.0005, \pm 0.002)$ & $1.307( \pm 0.002, \pm 0.001)$ \\
\hline & $216.2 \pm 0.3$ & $1.6347( \pm 0.0007, \pm 0.002)$ & N/A \\
\hline & $308 \pm 2$ & $1.6289( \pm 0.0007, \pm 0.002)$ & N/A \\
\hline & $407 \pm 1$ & $1.6229( \pm 0.0008, \pm 0.002)$ & N/A \\
\hline & $569 \pm 1$ & $1.617( \pm 0.002, \pm 0.002)$ & N/A \\
\hline
\end{tabular}

N/A, not applicable. 
Table S7. $K_{0}$ Values for Second Monomer Peak, Corresponding to [IsoF• $\left.\mathrm{CO}_{3}\right]^{-}$, on IMS Faraday Plate at 280.1 V/cm as a Function of Drift Gas Water Concentration at Three Drift Gas Temperatures under Undoped Conditions

\begin{tabular}{|c|c|c|}
\hline $\begin{array}{c}\text { Average } \\
\text { Drift Gas } \\
\text { Temperature } \\
\left({ }^{\circ} \mathbf{C}\right)\end{array}$ & $\begin{array}{c}\text { Average } \\
\text { Drift Gas } \\
\text { Water } \\
\text { Concentration } \\
\left(\mathbf{p p m}_{\mathbf{v}} \mathbf{H}_{\mathbf{2}} \mathbf{O}\right)\end{array}$ & $\begin{array}{c}\boldsymbol{K}_{\mathbf{0}} \text { Value } \\
(\text { Precision, Accuracy) } \\
\left(\mathbf{c m}^{\mathbf{2}} \mathbf{V}^{-\mathbf{1}} \mathbf{s}^{-\mathbf{1}}\right)\end{array}$ \\
\hline $30.02 \pm 0.03$ & $0.99 \pm 0.02$ & $1.612( \pm 0.002, \pm 0.002)$ \\
\cline { 2 - 3 } & $1.32 \pm 0.01$ & $1.614( \pm 0.002, \pm 0.002)$ \\
\hline $40.00 \pm 0.03$ & $1.00 \pm 0.01$ & $1.615( \pm 0.001, \pm 0.002)$ \\
\hline $50.01 \pm 0.04$ & $1.06 \pm 0.02$ & $1.619( \pm 0.002, \pm 0.002)$ \\
\hline
\end{tabular}

Table S8. $K_{0}$ Values for Second Monomer Peak, Corresponding to [IsoF• $\left.\mathrm{CO}_{3}\right]^{-}$, on IMS Faraday Plate at 280.2 V/cm as a Function of Drift Gas Water Concentration at Three Drift Gas Temperatures under Ammonia-Doped Conditions

\begin{tabular}{|c|c|c|}
\hline $\begin{array}{c}\text { Average } \\
\text { Drift Gas } \\
\text { Temperature } \\
\left({ }^{\circ} \mathbf{C}\right)\end{array}$ & $\begin{array}{c}\text { Average } \\
\text { Drift Gas } \\
\text { Water } \\
\text { Concentration } \\
\left(\mathbf{p p m}_{\mathbf{v}} \mathbf{H}_{\mathbf{2}} \mathbf{O}\right)\end{array}$ & $\begin{array}{c}\boldsymbol{K}_{\mathbf{0}} \text { Value } \\
(\text { Precision, Accuracy) } \\
\left(\mathbf{c m}^{\mathbf{2}} \mathbf{V}^{-1} \mathbf{s}^{-1}\right)\end{array}$ \\
\hline $30.07 \pm 0.03$ & $0.99 \pm 0.08$ & $1.612( \pm 0.001, \pm 0.002)$ \\
\hline $40.04 \pm 0.02$ & $0.955 \pm 0.004$ & $1.6176( \pm 0.0009, \pm 0.002)$ \\
\hline $50.00 \pm 0.03$ & $1.23 \pm 0.01$ & $1.623( \pm 0.002, \pm 0.002)$ \\
\hline
\end{tabular}

